\title{
METHODS, DIFFICULTIES, AND FIRST RESULTS IN LABORATORY SIMULATION OF COSMIC DUST ELECTRIC CHARGING
}

\author{
J. SVESTKA ${ }^{1}$ and E. GRÜN ${ }^{2}$ \\ ${ }^{2}$ Prague Observatory, Petrin 205, 11846 Prague 1, Czechoslovakia \\ ${ }^{2}$ Max-Planck-Institut für Kernphysik, 6900 Heidelberg 1, Germany
}

\begin{abstract}
Particles of radii 0.2 to $3 \mu \mathrm{m}$ and of different materials were suspended in an electrodynamic quadrupole inside a vacuum chamber and exposed to beams of electrons and ions of energies up to $20 \mathrm{keV}$ and $5 \mathrm{keV}$, respectively, with the aim to simulate electric charging of cosmic dust particles. It was found that the equilibrium surface electrostatic potential of glass particles of radii 0.2 to $2 \mu \mathrm{m}$ charged by electrons of energies 1 to $20 \mathrm{keV}$ is always positive. This can be explained by secondary electron emission at lower energies of electrons and by penetration of electrons through particles with subsequent secondary electron emission mainly from the exit side at higher energies. In case of charging by ions electrostatic potential of particles is generally much lower than expected values and interpretation of results of measurement is more complicated. The most promising way to eliminate instrumental influences disturbing processes of charging seems to be a construction of a smaller suspension system in which these influences would be negligible. Parameters of such a suspension system were derived from results of measurements.
\end{abstract}

\section{Introduction}

There are many phenomena connected to dust particles within the solar system which can be explained by their electric charging with subsequent interactions with electromagnetic fields and/or disruption by repulsive electrostatic forces - see e.g. Grün et al. (1984), Morfill et al. (1986), Boehnhardt and Fechtig (1987). Cosmic dust particles can be charged by a variety of mechanisms - see e.g. review of Whipple (1981). In case of solar system dust particles the most important charging processes are interactions with electrons and ions and photoemission by solar UV radiation. Theoretical calculations of electric charging by these processes are, however, especially in case of very small particles based on unreliable data extrapolated from results of measurements with plane surfaces (parameters of secondary electron emission and photoemission, capture probabilities of electrons and ions). Therefore we started experimental laboratory work on simulation of cosmic dust electric charging by electrons, ions and UV radiation - determination of equilibrium electrostatic surface potentials of particles of various sizes and materials exposed to beams of electrons and ions of different energies or illuminated by UV radiation of specific spectrum and intensity.

A.C. Levasseur-Regourd and H. Hasegawa (eds.), Origin and Evolution of Interplanetary Dust, 367-370. C 1991 Kluwer Academic Publishers, Printed in Japan. 


\section{Methods}

For charging of dust particles we used an electrodynamic quadrupole (minimum radius $r_{0}=25 \mathrm{~mm}$ ) inside a vacuum chamber (pressure down to $5.10^{-7} \mathrm{mbar}$ ) in which particles of radil 0.2 to $3 \mu \mathrm{m}$ and of various materials (glass, carbon, tungsten etc.) were suspended and exposed to electron or ion $\left(\mathrm{He}, \mathrm{Ar}, \mathrm{H}_{2}\right)$ beams of energies up to $20 \mathrm{keV}$ and $5 \mathrm{keV}$, respectively. The particles were illuminated by a HeNe laser and the light scattered by particles was observed by a telescope. $A$ charge-to-mass ratio $\mathrm{Q} / \mathrm{M}$ of a particle was determined from the amplitude of the quadrupole voltage $V$, applied frequency $f$, and oscillation frequency of a particle in vertical direction $f_{z}$ (determined with heip of a photomultiplier) by the formula

$$
\mathrm{Q} / \mathrm{M}=\text { const. } \mathrm{r}_{0}^{2} \mathrm{Hf}_{\mathrm{z}} / \mathrm{V}
$$

The charge $Q$ of the particle was found out by means of the charge induced on a metallic cylinder placed below the quadrupole through which the particle is forced to pass after completion of a charging process. From $Q / M$ and $Q$ the radius of the particle and its equilibrium surface electrostatic potential $U$ was calculated. Schematic diagram of the vertical cross section of the suspension system is shown in Fig. 1 - for more details about the suspension system see Svestka et al. (1987) or Pinter et al. (1990) and references therein. A similar suspension system in which particles will be suspended and charged by photoemission due to UV radiation produced by a deuterium lamp with $\mathrm{MgF}_{2}$ window is under construction.

The values of the equilibrium potential $U$ can be influenced by a presence of a rest gas in a vacuum chamber and by the electric field inside the quadrupole. In order to quantitatively characterize these influences dependencies of the equilibrium electrostatic surface potential $U$ on the vacuum pressure $p$ and on the amplitude of the quadrupole voltage $V$ for different energies of electrons and ions were studied.

\section{Results}

Test measurements were performed with lons because in absence of any disturbing instrumental influences equilibrium potential $U$ (in volts) should be simply equal to the energy of ions $E_{1}$ (in $\mathrm{eV}$ ) so that it is easy to compare results of measurements with ideal values. It was found that $U$ is increasing with decreasing $V$ and it is practically constant if $V$ is lower than approximately $E_{i} / 2$. $U$ is generally increasing with

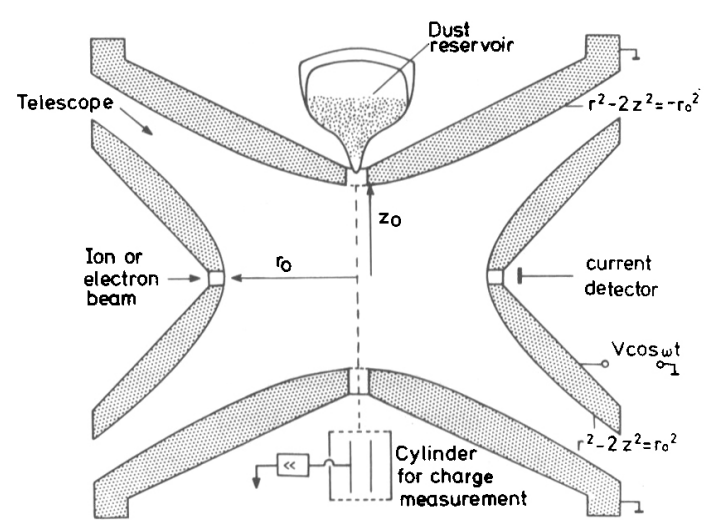

Figure 1. The vertical cross section of the particle suspension system. 
decreasing $p$ but within the energy range of charging ions it is always much lower than $E_{i}$ even for the lowest vacuum pressure. From the analysis of dependencies $U=U(p)$ and charging curves (dependencies of $U$ on time during charging) it was possible to derive an expression for the charging rate which contains the term corresponding to primary ions, the term due to electrons produced by collisional ionization of a rest gas which is proportional to vacuum pressure but also the third term which is independent of vacuum pressure and proportional only to ion current. This term is responsible for substantial lowering of $U$ below the ideal values and it is probably caused either by secondary electron emission due to lon impacts from the inner surfaces of the quadrupole electrodes or by field ionization of water molecules adsorbed on the surface of

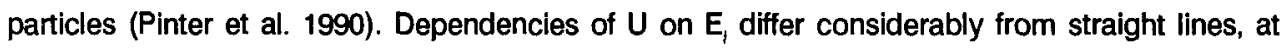
$E_{i}>2 \mathrm{keV}$ the potential $U$ is even decreasing with increasing $E_{i}$ - see Fig. 2.

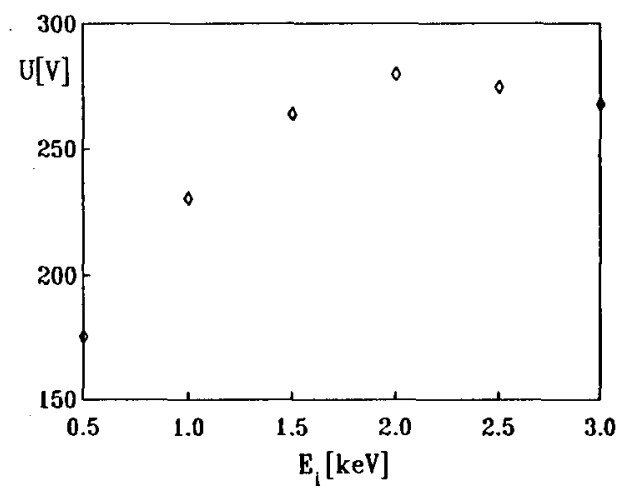

Figure 2. Typical dependence of particle equilibrium potential on energy of ions (glass particle of radius $0.47 \mu \mathrm{m}, \mathrm{p}=10^{-6}$ mbar, $\mathrm{He}^{+}$ions).

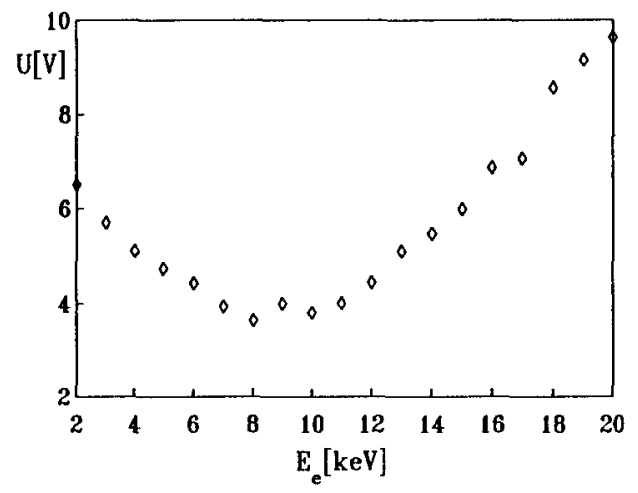

Figure 3. Typical dependence of particie equilibrium potential on encrgy of electrons (glass particle of radius $1.12 \mu \mathrm{m}, \mathrm{p}=10^{-6} \mathrm{mbar}$

Then we charged dust particles by electrons and studied analogously dependencies of $U$ on $V$, $p$ and $E_{e}$. With glass particles of radii up to $2 \mu \mathrm{m}$ and electrons of energies 1 to $20 \mathrm{keV}$ always positive particle potentials were found. $U$ is decreasing with increasing $E_{a}$ at lower $E_{0}$ due to decreasing efficiency of secondary electron emission but then increasing due to electrons penetrating through particles and causing secondary electron emission from the exit side - see Fig. 3. From the analysis of dependencies $U=U(p)$ and charging curves the expression for the charging rate was derived as in case of charging by ions. This expression contains again three terms corresponding respectively to primary electrons, secondary electrons (true secondaries and backscattered ones), and electrons produced by collisional ionization of a rest gas. The last term is responsible for measured potentials being lower compared to real "cosmic" ones and ensures that measured values give the lower limit to real potentials. 


\section{Discussion}

From preliminary results of electric charging by electrons it follows that the equilibrium electrostatic surface potential of glass particles of radii smaller then $2 \mu \mathrm{m}$ charged by electrons of energies 1 to $20 \mathrm{keV}$ is always positive. One should get the same result in case of water ice particles because the secondary electron emission yleld of glass and water ice is comparable - see Hashimov and Tarakanov (1982). This conclusion has already implications for solar system dust particles, e.g. for particles in planetary magnetospheres where electrons of energies 1 to $20 \mathrm{keV}$ are present. It was assumed that dust particles exposed to electrons of these energies will be generally charged to high negative potentials equal approximately to energy of electrons (in eV) - see e.g. Grün et al. (1984). On the contrary to that it follows from our results that it could be that only the potential of larger particles is high and negative but the potential of particles of micron and submicron sizes is much lower and it is positive.

A quantitative determination of the terms lowering values of $U$ is, however, rather difficult in cases of both electrons and ions. From equation (1) it follows that $V$ is proportional to $r_{0}^{2}$ for constant $Q / M$. To eliminate the influence of the electric field inside a quadrupole it is necessary to reduce the dimensions of the present quadrupole by a factor of 5 to 10 , i.e. to $r_{0}=2.5$ to $5 \mathrm{~mm}$. From the analysis of dependencies $U=U(p)$ and also from theoretical estimates it follows that the elimination of the influence of the rest gas requires $p \leq 10^{-10}$ mbar. The combination of low pressure with shorter path lengths of electrons and ions should make it possible to charge particles to equilibrium potentials without any collision of electrons or ions with an atom or molecule of a rest gas. For future work the construction of a smaller vacuum chamber, in which it will be possible to reach $p=10^{-10} \mathrm{mbar}$ and with a smaller quadrupole inside, seems to be the most promising possibility.

\section{References}

Boehnhardt, H. and Fechtig, H. (1987) 'Electrostatic charging and fragmentation of dust near P/Giacobini-Zinner and P/Halley', Astron. Astrophys. 187, 824-828.

Grün, E., Morfill, G. E. and Mendis, D. A. (1984) 'Dust-magnetosphere interactions', in R. Greenberg and A. Brahic (eds.), Planetary Rings, Univ. of Arizona Press, Tuscon, pp. 275-332.

Hashimov, N. M. and Tarakanov, V. L. (1982) 'Surface charge influence on sublimation rates of icy grains and cometary nuclei', Komety i Meteory 32, 3-9.(in Russian)

Morfill, G. E., Grün, E. and Leinert, C. (1986) 'The interaction of solid particles with the interplanetary medium', in R. G. Marsden (ed.), The Sun and the Heliosphere in Three Dimensions, D. Reidel Publishing Co., Dordrecht, pp. 455-474.

Pinter, S., Svestka, J. and Grün, E. (1990) 'Interaction of dust particles with electrons and ions', in E. Bussoletti and A. A. Vittone (eds.), Dusty Objects in the Universe, Kluwer Academic Publishers, Dordrecht, pp. 139-146.

Svestka, J., Grün, E., Pinter, S. and Schumacher, S. (1987) 'Laboratory charging of dust by electrons and ions', Publ. of Astron. Inst. of Czechoslovak Academy of Sci. 67, 277-280.

Whipple, E. C. (1981) 'Potentials of surfaces in space', Rev. Prog. Phys. 44, 1197-1250. 